\title{
ESTUDIO QUÍMICO Y MORFOLÓGICO DE RAMALINA POLYMORPHA GREX EN ESPAÑA
}

\author{
Rosario ARROYO, Estela SERIÑÁ \& Esteban MANRIQUE
}

\begin{abstract}
RESUMEN: La variabilidad morfológica que presenta Ramalina polymorpha grex, unida a la transición de caracteres entre algunos de los taxones del grupo, hace a veces dificultosa su determinación. Un amplio estudio químico y morfológico del grupo apoyado en su distribución y ecología, nos sugiere la presencia de dos taxones claramente diferenciables: Ramalina polymorpha (Ach.) Ach. y Ramalina capitata (Ach.) Nyl., incluyendo en ésta última el resto de las variaciones morfológicas encontradas en la Península Ibérica, que se tratan como variedades.
\end{abstract}

Palabras clave: líquenes, Ramalina, quimiotaxonomía, España.

ABSTRACT: The identification of members of the Ramalina polymorpha grex is often complicated by the considerable morphological variation and intergradation of the characters within the group. Based on a broad chemical and morphological study of this group and its ecology and distribution, we recognize two distinct taxa: Ramalina polymorpha (Ach.) Ach. and Ramalina capitata (Ach.) Nyl., the latter including the rest of the morphological variations encountered on the Iberian Peninsula, wich are treated here as varieties.

Key words: lichens, Ramalina, chemotaxomomy, Spain.

\section{INTRODUCCIÓN}

Una de las características de las especies que conforman el grupo Ramalina polymorpha es su alta variabilidad morfológica; esto ha sido constatado por diversos autores desde Acharius (1810) hasta la actualidad, en que el problema a dilucidar, esto es, decidir si hay o no rango específico en estas variaciones sigue en discusión. Dentro del concepto de esta especie se incluyen taxones como: Ramalina polymorpha (Ach.) Ach., $R$. capitata (Ach.) Nyl., $R$. protecta H. Magn., R. strepsilis (Ach.) Zahlbr. y $R$. digitellata Nyl., que en algunos casos se diferencian bien morfológicamente.

El problema que se presenta es cómo interpretar la transición de caracteres entre estos taxones, que aparece en un elevado número de ejemplares, lo que ha llevado a una diversidad de opiniones entre los autores que han estudiado este grupo de especies. Krog \& James (1977), Krog \& Osthagen (1980), constatan la gran diversidad de formas que caracteriza al grupo Ramalina polymorpha y excluyen a $R$. 
capitata y formas relacionadas, refiriéndose a diferentes morfótipos de una única especie Ramalina polymorpha (Ach.) Ach. Santesson (1984) reconoce dos especies claramente diferenciables: Ramalina capitata (Ach.) Nyl., y $R$. polymorpha (Ach.) Ach., constatando que $R$. capitata está bien separada de $R$. polymorpha. L.G. Sancho (1986. Tesis Doctoral inédita) encuentra en el Sistema Central español dos morfologías claramente asimilables a especies: Ramalina polymorpha y $R$. capitata, destacando que $R$. polymorpha es más abundante en la alta montaña mediterránea, ligada a ciertos hábitats en las rocas muy expuestas y conviviendo con formas bien diferenciadas de R. capitata. Nimis \& Poelt (1987) reconocen también únicamente dos especies: Ramalina capitata y $R$. polymorpha, subordinando $R$. strepsilis como variedad de $R$. capitata y $R$. protecta y $R$. digitellata como variedades de $R$. polymorpha.

El objetivo del presente trabajo es contribuir a la caracterización de este grupo de especies en la Península Ibérica, basándonos en su composición química (sustancias liquénicas), morfología, ecología y distribución.

\section{MATERIAL Y MÉTODOS}

Se han estudiado morfológica y químicamente 891 ejemplares procedentes de 52 localidades españolas, todos ellos recogidos sobre rocas ácidas. Los pliegos correspondientes quedan depositados en el herbario MAF.

Los análisis químicos se han realizado mediante las técnicas de cromatografía en capa fina (TLC) y cromatografía líquida de alta resolución (HPLC). Para TLC se ha seguido a Culberson \& Kristinsson (1970) y Culberson (1972,1974), utilizando los disolventes A,B,C y placas de silicagel 60F 254 (Merck 5554). Como controles se llevaron en todas las placas: atranorina (Sigma Chem. Co.) y ácido norestíctico (extracto acetónico de Parmelia acetabulum (Neck.) Duby). Después de analizar los 891 ejemplares por TLC, se seleccionaron 3 ó 4 talos de cada una de las especies del grupo, para ser analizadas por HPLC, siguiendo la metodología utilizada por Arroyo \& Manrique $(1988,1989)$. Se ha utilizado en todos los casos como fase móvil, metanol-agua-ácido acético (75:25:2), y una columna de $25 \mathrm{~cm} \times 4.6 \mathrm{~mm}, 5 \mu \mathrm{m}$ Spherisorb ODS-2. Las sustancias utilizadas en este caso como patrones han sido: ácido usneico (Sigma Chem. Co.) y ácido norestíctico (extracto acetónico de Parmelia acetabulum ), con un tiempo de retención de 29.23 y 5.51 minutos respectivamente.

Con objeto de estudiar la distribución de las sustancias liquénicas a lo largo de las lacinias del talo, se seleccionó una ramita de cada muestra, y se dividió en tres fragmentos ( $\mathrm{A}=$ zona basal, $\mathrm{B}=$ zona media y $\mathrm{C}=$ zona apical $)$ de los que se obtuvo el peso seco a $100{ }^{\circ} \mathrm{C}$ en estufa durante 24 horas, expresándose los resultados en unidades de área de pico por miligramo de peso seco.

\section{RESULTADOS Y DISCUSIÓN}

El análisis morfológico de todos los ejemplares recogidos revela la existencia 
de una serie de caracteres más o menos claramente diferenciados: pseudocifelas laminales, superficie irregular de las lacinias y soredios granulosos. La localización de los soralios, sin embargo, es el carácter que mejor permite la diferenciación entre los distintos taxones que conforman este grupo (Fig. 1).
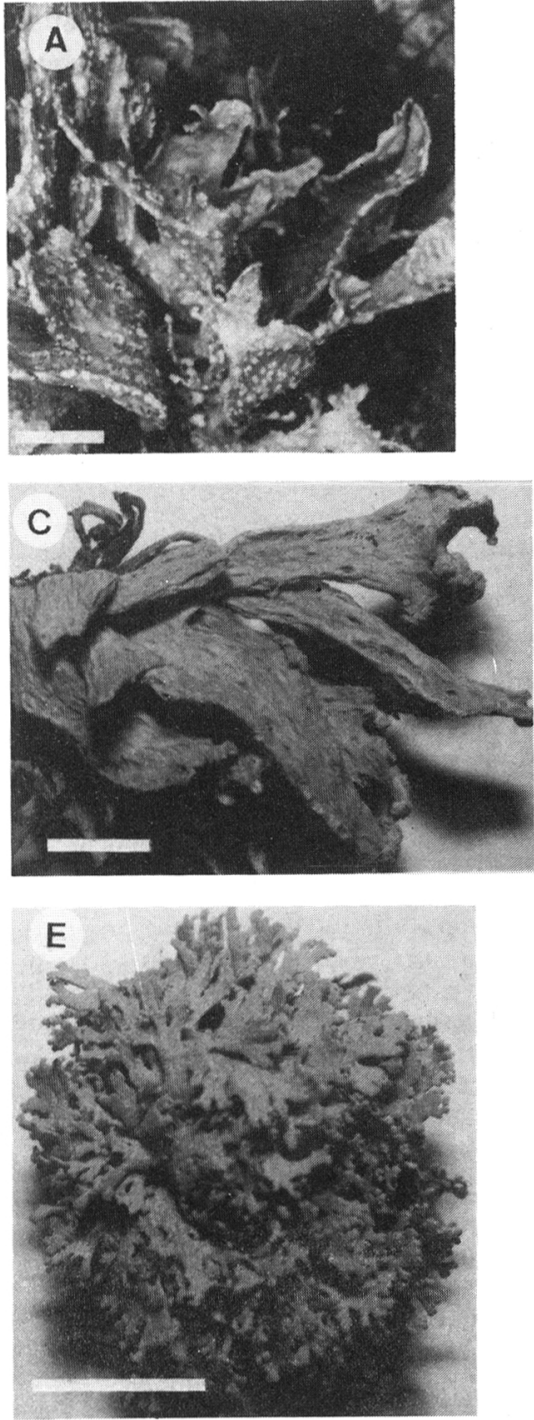
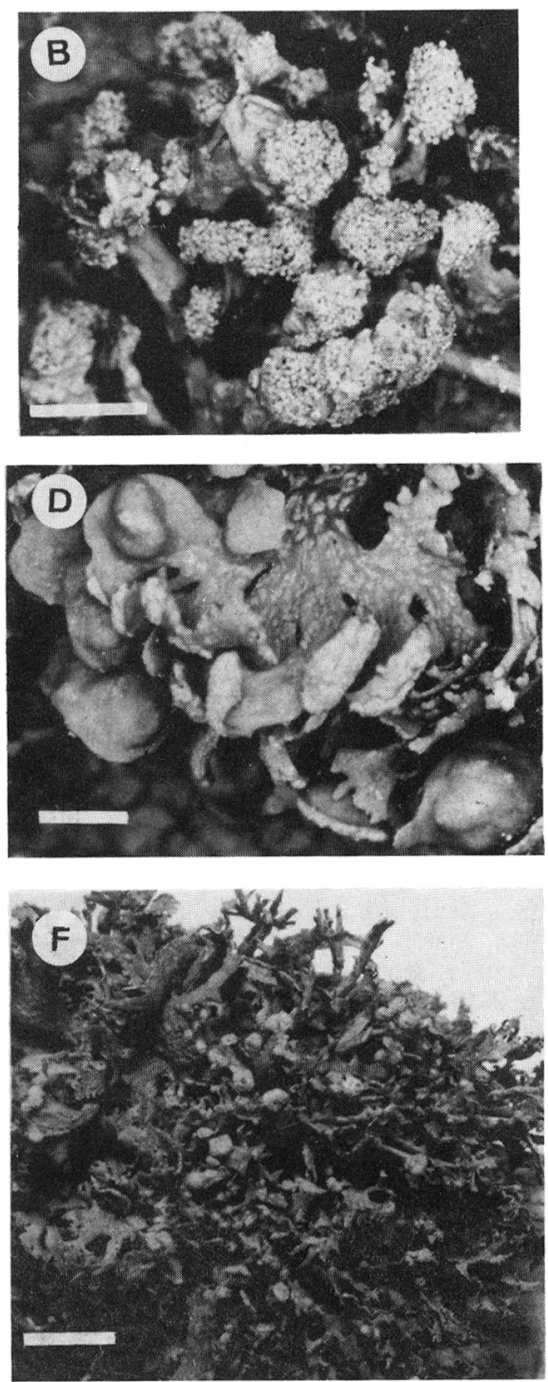

Fig. 1.- Aspecto del talo y tipos de soralios presentes en Ramalina polymorpha grex: A) Ramalina polymorpha. s. str. (laminales); B) R. capitata (capitiformes); C) R. strepsilis (capitiformes y la briformes); D) R.protecta (forniciformes); E) R. digitellata (soredios debajo de ramificaciones digitiformes); F) morfología intermedia. (regleta $=5 \mathrm{~mm}$ ) 
Ramalina polymorpha s. str. presenta soralios laminales que derivan de pseudocifelas, con gránulos corticados (soredios granulosos), mientras que en el resto de los taxones los soralios tienden a ser terminales y subterminales: $R$. capitata, capitiformes; $R$. strepsilis, capitiformes y labriformes; $R$. protecta, forniciformes, y $R$. digitellata, con ramificaciones digitiformes características en el extremo de las lacinias, que se curvan débilmente hacia su cara inferior, encontrándose los soredios debajo de éstas.

Los principales caracteres morfológicos observados, número de talos analizados, rango altitudinal de las localidades en que cada taxon ha sido recogido y sustrato, quedan reflejados en la tabla 1 . Se ha comprobado, que si bien hay especímenes claramente diferentes entre sí, pudiendo ser asimilados cada uno de ellos a taxones independientes, (Ramalina polymorpha $3.14 \%, R$. capitata $19.4 \%, R$. strepsilis $3.36 \%, R$. protecta $27.16 \%, R$. digitellata $24 \%$ ), existe un elevado número de ejemplares $(21.66 \%)$, que presentan caracteres intermedios entre $R$. capitata, $R$. digitellata y $R$. protecta $($ Fig. 1F), lo que apoyaría el criterio sintético final adoptado por Krog \& James (o.c.). Sin embargo, no hemos detectado transición de caracteres entre estos taxones y $R$. polymorpha s. str..

\begin{tabular}{lccccccccccccccccc}
\hline & 1 & 2 & 3 & 4 & 5 & 6 & 7 & 8 & 9 & 10 & 11 & 12 & 13 & 14 & 15 & 16 \\
\hline $\begin{array}{l}\text { R. polymorpha } \\
\text { R. capitata }\end{array}$ & 28 & + & + & + & + & + & 6 & 4 & - & - & - & - & $(28)$ & - & $1600-2500$ & + & - \\
R. strepsilis & 30 & + & + & + & - & 2 & 3 & + & + & - & - & $(154)$ & $(19)$ & $100-2500$ & + & + \\
R. protecta & 242 & + & + & + & - & 3 & 4 & + & + & - & - & $(21)$ & $(9)$ & $100-2500$ & + & + \\
R. digitellata & 214 & + & + & + & - & 2 & 3 & - & - & + & - & $(210)$ & $(32)$ & $80-2500$ & + & + \\
Morfología & 193 & + & + & + & - & 2 & 3 & \pm & \pm & \pm & + & $(193)$ & $(21)$ & $80-2500$ & + & + \\
intermedia & & & & & & & & & & & & & & & & & \\
\hline
\end{tabular}

Tabla 1. Principales caracteres morfológicos, rango altitudinal, sustrato y composición química (sustancias liquénicas) en Ramalina polymorpha Grex. Entre paréntesis se indica el $\mathrm{n}^{\mathrm{o}}$ de talos analizados que presentan solamente ácido usneico, o bien, ambos ácidos: usneico y norestíctico; $1: \mathrm{n}^{\mathrm{o}}$ talos; 2 : ps. lam.= pseudocifelas laminales; 3 : sup. irreg. $=$ superficie irregular; 4: sor. gran.= soredios granulosos; 5 : gran. cort.= pseudocifelas con gránulos corticados; 6: long.= longitud máxima de las lacinias en $\mathrm{cm} ; 7$ : anch.= anchura máxima de las lacinias en mm; 8: soral. cap.= soralios capitiformes; 9: soral. lab.= soralios labriformes; 10 : soral. for.= soralios forniciformes; 11 : ex. dig.= ramificaciones extremas digitiformes; 12 : usneico; 13: norest.= ácido norestíctico; 14: altitud; 15 : $r$. ex.= rocas expuestas; 16: r. prot.= rocas protegidas.

Los análisis químicos realizados por TLC en los 891 talos revelan como componente mayoritario y siempre presente el ácido usneico, única sustancia detectada por Krog \& James (o.c.), Krog \& Osthagen (o.c.). En algunos ejemplares (11.45\%), siempre recogidos en localidades inferiores a los $2000 \mathrm{~m}$ de altitud, además se presenta el ácido norestíctico, causante de la reacción $\mathrm{K}+$ rojo en médula y soralios; la presencia esporádica de dicha sustancia, se ha detectado en todas las morfologías 
descritas excepto en aquellos ejemplares que entrarían en el concepto de Ramalina polymorpha s. str. (tabla 1). En el $34.56 \%$ de los talos analizados se observa una mancha correspondiente a una sustancia no identificada (SDP), cuyas características cromatográficas y reacciones de coloración se presentan en la tabla 2. Esta sustancia aparece esporádicamente en todas las morfologías descritas junto con el ácido usneico, pero nunca con el ácido norestíctico y sólo en una ocasión se ha detectado en Ramalina polymorpha s. str.

\begin{tabular}{|c|c|c|c|c|c|c|}
\hline \multirow[b]{2}{*}{ Sustancia } & \multirow[b]{2}{*}{ A } & \multicolumn{2}{|l|}{$\operatorname{Rf} \times 100$} & \multicolumn{2}{|c|}{ Clase Rf } & \multirow[t]{2}{*}{ Coloración } \\
\hline & & $\mathrm{B}$ & $\mathrm{C}$ & A B & $\mathrm{C}$ & \\
\hline ác. usneico & $65 / 47.66$ & $64 / 37.71$ & $58 / 28.70$ & 066 & 6 & gris verdoso $0^{\circ}$ \\
\hline ác. norestíctico & $47 / 47.66$ & $37 / 37.71$ & $28 / 28.70$ & $\begin{array}{lll}0 & 4 & 4\end{array}$ & 4 & amarillo $^{\circ}, \mathrm{PD}+$ amarillo, $\mathrm{K}+$ rojo \\
\hline SDP & $26 / 47.66$ & $15 / 37.71$ & $8 / 28.70$ & 32 & 2 & gris amarillento $^{\circ}$ \\
\hline
\end{tabular}

Tabla 2. Características cromatográficas y reacciones de coloración de las sustancias liquénicas detectadas por TLC. $*$ Coloración tras el revelado con $\mathrm{SO}_{4} \mathrm{H}_{2} 10 \%$ en agua, con posterior calentamiento a $110^{\circ} \mathrm{C} ; \mathrm{SDP}=$ sustancia no identificada.

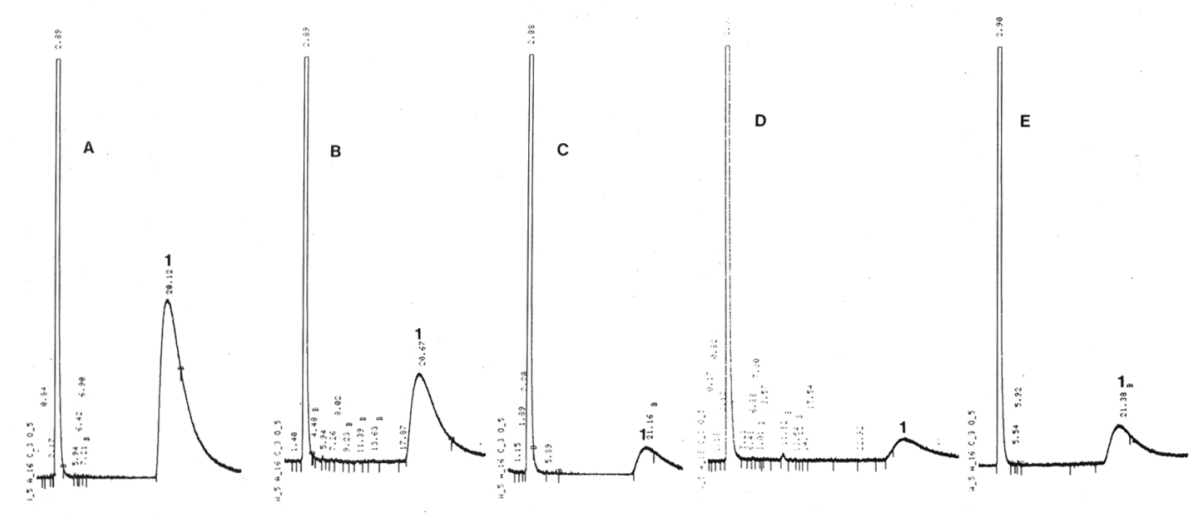

Fig. 2.- Cromatogramas correspondientes a los análisis realizados por HPLC, con el ácido usneico como único componente: A) Ramalina polymorpha s. str.; B) R. capitata; C) R. digitellata; D) R. protecta; E) Morfología intermedia; 1 = ácido usneico.

En los análisis por HPLC, realizados en una selección de ejemplares correspondientes a cada uno de los taxones (Figs. 2 y 3), se observa en todos los casos un pico mayoritario correspondiente al ácido usneico, de concentración variable en diferentes talos de una misma especie así como en las distintas zonas de un mismo talo (tabla 4); la concentración se expresa en unidades arbitrarias de área de pico/ miligramo de peso seco del líquen, correspondiendo el valor mínimo a la zona basal 
(más vieja), y el valor máximo a la zona apical (más jóven y en general más cargada de soredios), (Fahselt, 1984; Geyer, 1985; López Redondo, 1988; Seriñá, 1990 Tesis Doctoral inédita). En algunos ejemplares asimilables a Ramalina capitata, R. strepsilis, $R$. protecta y $R$. digitellata, así como en algunos que presentan caracteres intermedios, se ha detectado un segundo pico correspondiente al ácido norestíctico, que si bien no está en concentración elevada, sí muesra una concentración creciente hacia las partes más jóvenes del talo, al igual que el ácido usneico. Dicha sustancia, sin embargo, está ausente en los ejemplares de Ramalina polymorpha s. str. confirmando nuestros resultados por TLC. De la misma manera, se observa un pico no identificado (SDH), presente en slgunos talos de toda las morfologías estudiadas, excepto en Ramalina polymorpha s. str.; este pico no parece corresponder a la sustancia desconocida (SDP) detectada por TLC, ya que algunos ejemplares que la presentan por esta técnica, no muestran el SDH al ser analizados por HPLC. Los tiempos de retención (valor medio) para cada una de las sustancias mencionadas, se presentan en la tabla 3.

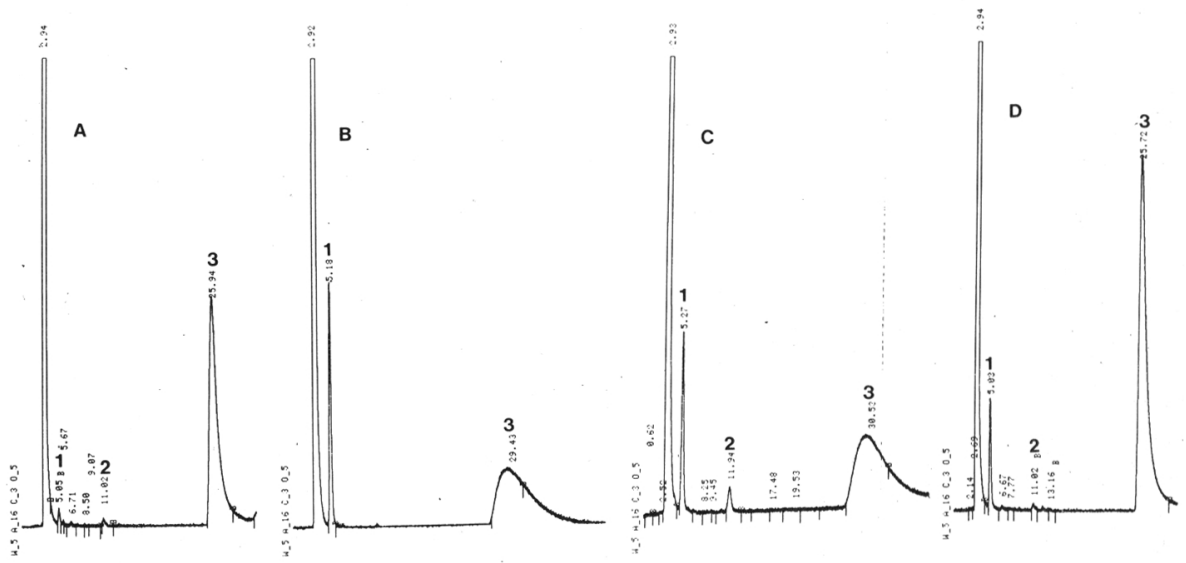

Fig. 3.- Cromatogramas correspondientes a los análisis realizados por HPLC, con presencia de los ácidos usneico y norestíctico: A) Ramalina capitata; B) R. digitellata; C) R. protecta; D) Morfología intermedia; $1=$ ácido norestíctico; $2=\mathrm{SDH}$ (pico no identificado); $3=$ ácido usneico.

\begin{tabular}{lc}
\hline Sustancia & tR $(75: 25: 1)$ \\
\hline ácido norestíctico & $5.12 \pm 0.08$ \\
SDH & $11.43 \pm 0.28$ \\
ácido usneico & $27.19 \pm 0.76$ \\
\hline
\end{tabular}

Tabla 3. Valor medio del tiempo de retención de cada una de las sustancias. SDH= pico no identificado; $\mathrm{t} R=$ tiempo de retención en minutos. 


\begin{tabular}{|c|c|c|c|c|c|c|c|c|c|c|}
\hline & Talos & $\mathrm{A}^{\mathrm{a}}$ & $\begin{array}{c}\text { B } \\
\text { ido ust }\end{array}$ & co & $\begin{array}{l}\text { A } \\
\text { ácid }\end{array}$ & $\begin{array}{c}\text { B } \\
\text { torest }\end{array}$ & $\begin{array}{r}\text { C } \\
\text { ico } \\
\end{array}$ & A & $\begin{array}{c}\mathrm{B} \\
\mathrm{SDH}\end{array}$ & $\mathrm{C}$ \\
\hline \multirow[t]{3}{*}{ R. polymorpha } & 1 & 4.9 & 17.0 & 22.7 & - & - & - & - & - & - \\
\hline & 2 & 0.0 & 5.4 & 20.0 & - & - & - & - & - & - \\
\hline & 1 & 0.0 & 0.0 & 11.8 & 0.0 & 0.0 & 0.0 & 0.0 & 0.0 & 0.0 \\
\hline \multirow[t]{3}{*}{ R. capitata } & 2 & 2.3 & 1.5 & 16.4 & 0.0 & 0.0 & 0.2 & 0.5 & 0.2 & 0.2 \\
\hline & 3 & 0.6 & 2.5 & 21.0 & 0.0 & 0.0 & 0.0 & 0.7 & 0.3 & 0.3 \\
\hline & 4 & 0.8 & 0.9 & 52.0 & 0.0 & 0.0 & 0.0 & 0.1 & 0.2 & 0.2 \\
\hline \multirow[t]{3}{*}{$R$. digitellata } & 1 & 0.0 & 0.0 & 4.5 & 0.1 & 1.0 & 3.5 & 0.2 & 0.0 & 0.0 \\
\hline & 2 & 0.0 & 0.0 & 3.0 & 0.0 & 0.0 & 0.0 & 0.0 & 0.0 & 0.0 \\
\hline & 1 & 0.0 & 0.0 & 5.9 & 0.8 & 0.4 & 1.9 & 0.2 & 0.3 & 0.5 \\
\hline \multirow[t]{2}{*}{ R. protecta } & 2 & 0.0 & 2.0 & 97.2 & 0.1 & 0.2 & 1.4 & 0.1 & 0.1 & 0.2 \\
\hline & 3 & 1.3 & 9.5 & 23.3 & 0.0 & 0.2 & 0.1 & 1.5 & 6.2 & 2.5 \\
\hline \multirow{2}{*}{$\begin{array}{l}\text { Morfología } \\
\text { intermedia }\end{array}$} & 1 & 0.0 & 0.0 & 3.3 & 0.0 & 0.0 & 0.0 & 0.0 & 0.0 & 0.0 \\
\hline & 2 & 2.3 & 0.7 & 11.8 & 0.2 & 0.5 & 0.9 & 0.2 & 0.2 & 0.1 \\
\hline
\end{tabular}

Tabla 4. Variación intratalina en la concentración de las sustancias detectadas por HPLC en Ramalina polymorpha grex. A, B, C: diferentes zonas del talo considerando una lacinia: A= basal; $\mathrm{B}=$ media; $\mathrm{C}=$ apical; ác. usn.= ácido usneico; ác. nor.= ácido norestíctico; $\mathrm{SDH}=$ pico no identificado.

Once de los ejemplares estudiados (1.28\%), presentan gran profusión de soralios en general no muy bien delimitados, capitiformes, forniciformes, marginales y algunos laminales; han sido recogidos en localidades comprendidas entre los 1500 y $1600 \mathrm{~m}$ de altitud, siempre en zonas protegidas de las rocas. Su morfología podría ser asimilable a Ramalina pollinaria (Westr.) Ach., pero esta especie siempre contiene en la médula ácido evérnico (Krog \& James, 1977; Arroyo \& Manrique, 1989), mientras que estos ejemplares solamente presentan ácido usneico, por lo que se han incluido en este grupo de especies.

Los talos asimilables a Ramalina polymorpha s. str. siempre se han recogido en localidades comprendidas entre $\operatorname{los} 1600$ y $2500 \mathrm{~m}$ (Fig.4), mientras que el resto de los taxones además de localizarse dentro de estos límites, también se han encontrado en localidades mucho más bajas, entre 100 y 1500 m (Figs. 5 y 6).

Ramalina polymorpha s. str. crece siempre sobre afloramientos rocosos muy expuestos, espolones de alta montaña, conviviendo con formas bien diferenciadas de $R$. capitata, mientras que el resto de las morfologías crecen tanto sobre zonas expuestas de rocas, como sobre paredes verticales que quedan resguardadas de la lluvia. Ramalina capitata muestra preferencia hacia un sustrato expuesto, mientras que $R$. propecta predomina sobre zonas de las rocas que quedan protegidas. $R$. digitellata, así como los ejemplares que muestran transición de caracteres, crecen indistintamente en ambos hábitats sin apreciarse una preferencia dominante. 


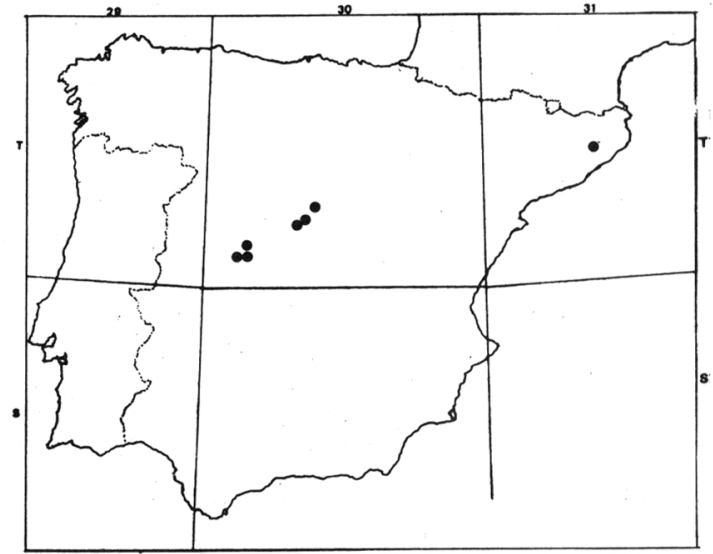

Fig. 4.- Localidades muestreadas en las que se ha encontrado Ramalina polymorpha s. str..
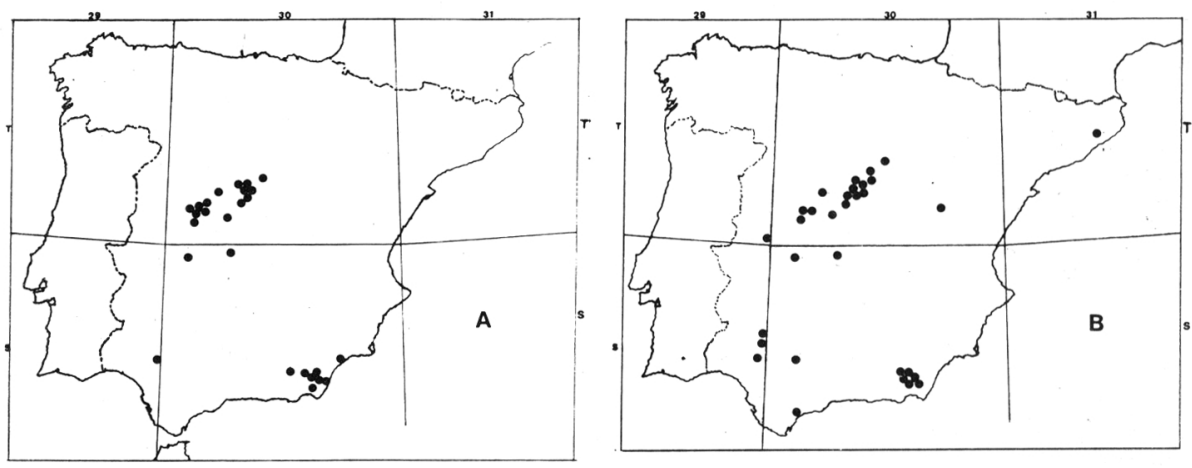

Fig. 5.- Localidades muestreadas en las que se ha encontrado: A) Ramalina capitata; B) $R$. digitellata.
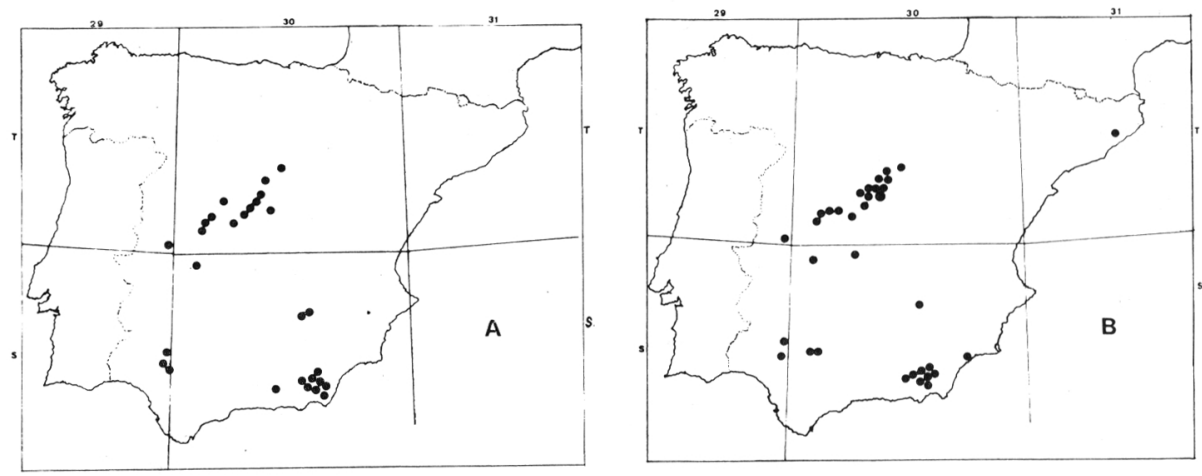

Fig. 6.- Localidades muestreadas en las que se ha encontrado: A) Ramalina protecta; B) Morfología intermedia. 


\section{CONCLUSIONES}

En base a todos los análisis realizados, pensamos que Ramalina polymorpha (Ach.) Ach. muestra unas características propias que la separan del resto de las morfologías estudiadas. Estas últimas, sin embargo, presentan similitudes con Ramalina capitata (Ach.) Nyl. tanto a nivel químico como morfológico y ecológico, por lo que sugerimos el mantenimiento de estos dos taxones, considerando el resto de las variaciones morfológicas como diferentes morfótipos de Ramalina capitata (Ach.) Nyl. a los que se podría asignar el rango de variedad.

\section{AGRADECIMIENTOS}

Este trabajo se ha realizado en el marco del Proyecto de Investigación subvencionado por la CAYCIT, n 2954/83 C0202.

\section{BIBLIOGRAFÍA}

ACHARIUS, E. -1810- Lichenographia universalis. Gottingae.

ARROYO-CABEZA, R. \& E. MANRIQUE-REOL -1988- Estudios químicos en Ramalina farinacea (L.) Ach. del centro de España. Anales Jard. Bot. Madrid 45 (1): 53-59.

ARROYO-CABEZA, R. \& E. MANRIQUE-REOL -1989- Estudios químicos del género Ramalina Ach. del centro de la Península Ibérica. Anales Jard. Bot. Madrid 46 (1): $307-$ 315.

CULBERSON, C.F. - 1972- Improved conditions and new data for the identification of lichen products by a standardized thin-layer chromatographic method. J. Chromatogr. 72: 113125.

CULBERSON, C.F. -1974- Conditions for the use of Merck silica gel 60F 254 plates in the standardized thin-layer chromtographic technique for lichen products. J. Chromatogr. 97 : 107-108.

CULBERSON, C.F. \& H. KRISTINSSON-1970- A standardized method for the identification of lichen products. J. Chromatogr. 46: 85-93.

FAHSELT, D. -1984- Interthalline variability in leves of lichen products within stands of Cladina stellaris. The Bryologist 87 (1): 50-56

GEYER, M. -1985- Hochdruck-Flüssigkeits-Chromatographie (HPLC) von FlechtenSkundärstoffen. 233 pp. Diss. Univ. Essen.

KROG, H. \& P.W. JAMES -1977- The genus Ramalina in Fenoscandia and the British Isles. Norw. J. Bot. 24: 15-43.

KROG, H. \& H. OSTHAGEN -1980- The genus Ramalina in the Canary Islands. Norw.J. Bot. 27: 255-296.

LOPEZ-REDONDO, F. -1988- Aportaciones al conocimiento fitoquímico de Pseudevernia furfuracea (L.) Zopf en España. Universidad Complutense de Madrid. Memoria de Licenciatura inédita.

NIMIS, P.L. \& J. POELT -1987- The lichens and lichenicolous fungi of Sardinia (Italy). Studia geobotanica an Internacional Journal 7, Supp. 1: 194-203.

SANTESSON, R. -1984- The lichens of Sweden and Norway. Stockholm and Upsala.

SANCHO, L.G. -1986- Flora y vegetación liquénica saxícola de los pisos oro- y crioromediterráneo del Sistema Central español. Facultad de Biología. Universidad Complutense de Madrid. Tesis Doctoral inédita. 
SERIÑA-RAMIREZ, E. -1990- Estudio quimiotaxonómico en líquenes de la provinvcia de Madrid. Facultad de Biología. Universidad Complutense de Madrid. Tesis Doctoral inédita.

\section{RELACION DE LOCALIDADES MUESTREADAS}

ALBACETE: Alcaraz, Reolid, Sa del Relumbrar, 100-1100 m, 30SWH27; Alcaraz, Reolid, S $\mathbf{a}$ del Relumbrar, Cañada del Conejo, 830 m, 30 SWH27.

ALMERIA: Uleila del Campo, S ${ }^{\mathrm{a}}$ de los Filabres, Monteagudo, $1300 \mathrm{~m}, 30 \mathrm{SWG} 7119$; Uleila del Campo, S de los Filabres, La Peñica, 1100 m, 30SWG71; Gérgal, Sª de los Filabres, Arroyo Verruga, 1900 m, 30SW40; Bacares, S de los Filabres, Barranco Barrancón, 1700 m, 30SWG42; Gérgal, $\mathrm{S}^{\mathrm{a}}$ de los Filabres, Barranco del Pino, $1850 \mathrm{~m}$, 30SWG40; $\mathrm{S}^{\mathrm{a}}$ de Filabres, Calar Alto, 1900-2000 m, 30SWG4020; Bacares, Sa de los Filabres, Collado del Conde, 1900 m, 30SWG42; Bacares, Sa de los Filabres, Hoya Merendera, 1500-1850 m, 30SWG4924; Mojácar, S de Cabrera, La Adelfa, 500 m, 30SWG9702; Tabernas, S" Alhamilla, Pico Alhamilla, 1365 m, 30SWG69; Gérgal, S de los Filabres, Piedra del Sombrero, 900 m, 30SWG40; Uleila del Campo, S ${ }^{\mathrm{a}}$ de los Filabres, Rambla del Pautí, 800 m, 30 SWG71.

AVILA: Cardeñosa, 1100 m, 30TUL5312; Cepeda La Mora, 1550 m, 30TUK2681; S de Gredos, Garganta del Circo de Gredos, 2050 m, 30TUK0762; S de Gredos, Garganta Cinco Lagunas, $1850 \mathrm{~m}$, 30TUK0363; La Serrota, $1950 \mathrm{~m}$, 30TUK2485; $\mathrm{S}^{\mathrm{a}}$ de Gredos, Navarredonda de Gredos, 1500 m, 30TUK1971; Sa de Gredos, Circo de Gredos, Risco Negro, 2300 m, 30TUK05; Sª de Gredos, San Martín del Pimpollar, 1500 m, 30TUK2671.

BARCELONA: S ${ }^{\mathrm{a}}$ de Montseny, Cresta de Les Agudes, 1600 m, 31 TDG5326.

CACERES: Montfrague, subida al castillo, $300 \mathrm{~m}$, 29SQE5413; Trujillo, subida al castillo, $500 \mathrm{~m}$, 30STJ5272.

CADIZ: Alcalá de los Gazules, S ${ }^{\mathrm{a}}$ del Aljibe, $800 \mathrm{~m}, 30 \mathrm{STF} 6445$.

GRANADA: Guadix, $S^{\text {a }}$ de Baza, Piedra del Deseo, $1900 \mathrm{~m}, 30 \mathrm{SWG} 22$; Guadix, $\mathrm{S}^{\mathrm{a}}$ de Baza, Padilla, 2000 m, 30SWG1925; Guadix, S de Baza, Umbría, 750 m, 30SWG22; S Nevada, Peñones de San Francisco, 2500 m, 30SVG6607.

MADRID: Collado Mediano, Monte de Roblepollo, 1000 m, 30TVL1305; Manzanares El Real, La Pedriza, pista forestal, 1400 m, 30TVL2611; Manzanares El Real, La Pedriza, al pié de cabezas de Hierro, 1900 m, 30TVL2117; Montejo de la Sierra, Hayedo de Montejo, 1300 m, 30TVL5545; Peña de Cenicientos, 1250 m, 30TUK 7359; Puerto de la Morcuera, 1550 m, 30TVL3119; San Lorenzo de El Escorial, Abantos, 1700 m, 30TVK0297; Colgadizos, 2000 m, 30TVL4653; $\mathrm{S}^{\mathrm{a}}$ de Guadarrama, Dos Hermanas, $2000 \mathrm{~m}$, 30TVL1922; $\mathrm{S}^{\mathrm{a}}$ de Guadarrama, La Peñota, $1600 \mathrm{~m}$, 30TVL4043; S $\mathrm{S}^{\mathrm{a}}$ de Guadarrama, Peñalara, $2430 \mathrm{~m}$, 30TVL1922; S ${ }^{\mathrm{a}}$ de Guadarrama, Valdesquí, Refugio El Pingarrón, 1800 m, 30TVL12; Soto de Viñuelas, $740 \mathrm{~m}$, 30TVK4995.

MURCIA: Aguilas, S ${ }^{\mathrm{a}}$ del Cantal, Tinajeros, 700 m, 30SXG2753.

SEVILLA: Almadén de la Plata, Cortijo El Berrocal, 450 m, 29SQB6193; Gerena, 100 m, 29SQB5258; Guillena, 80 m, 29SQB5959; Lora del Río, Chaparras de Doña Rosa, 220 m, 30STG7575; Lora del Río, S a de la Cruz, $150 \mathrm{~m}$, 30STG7473; Sª Padrona, El Real de la Jara, $600 \mathrm{~m}, 29 \mathrm{SQC} 6102$.

SORIA: Tiermes, $1200 \mathrm{~m}, 30$ TVL 8479.

TERUEL: Orihuela de El Tremedal, El Tremedal, $1500 \mathrm{~m}, 30 \mathrm{TXK} 18$.

TOLEDO: San Pablo de los Montes a Menasalbas, 700 m, 30 SUJ88.

(Aceptado para su publicación en Junio de 1.990)

Dirección de los autores: R. ARROYO \& E. SERIÑÁ: Dpto. de Biología Vegetal I, Facultad de Biología, Universidad Complutense. 28040 Madrid. E. MANRIQUE: Dpto. de Biología Vegetal II, Facultad de Farmacia, Universidad Complutense. 28040 Madrid. 